\title{
The Human Lumbar Intervertebral Disc \\ Evidence for Changes in the Biosynthesis and Denaturation of the Extracellular Matrix with Growth, Maturation, Ageing, and Degeneration
}

\author{
John Antoniou, ${ }^{\star}$ Thomas Steffen, ${ }^{*}$ Fred Nelson, ${ }^{\ddagger}$ Neil Winterbottom, ${ }^{\S}$ Anthony P. Hollander, ${ }^{\ddagger}$ Robin A. Poole, ${ }^{\ddagger}$ Max Aebi, ${ }^{\star}$ \\ and Mauro Alini* \\ *Orthopaedic Research Laboratory, Royal Victoria Hospital, and Division of Orthopaedic Surgery, McGill University, Montreal, Quebec, \\ H3A 1 A1 Canada; ${ }^{\ddagger}$ Joint Diseases Laboratory, Shriners Hospital for Crippled Children, and Division of Orthopaedic Surgery, McGill \\ University; and ${ }^{\S}$ Metra Biosystems, Inc., Palo Alto, California, 94043
}

\section{Abstract}

Very little is known about the turnover of extracellular matrix in the human intervertebral disc. We measured concentrations of specific molecules reflecting matrix synthesis and degradation in predetermined regions of 121 human lumbar intervertebral discs and correlated them with ageing and Thompson grade of degeneration.

Synthesis in intervertebral discs, measured by immunoassay of the content of a putative aggrecan biosynthesis marker (846) and the content of types I and II procollagen markers, is highest in the neonatal and 2-5-yr age groups. The contents of these epitopes/molecules progressively diminished with increasing age. However, in the oldest age group (60-80 yr) and in highly degenerated discs, the type I procollagen epitope level increased significantly.

The percentage of denatured type II collagen, assessed by the presence of an epitope that is exposed with cleavage of type II collagen, increased twofold from the neonatal discs to the young 2-5-yr age group. Thereafter, the percentage progressively decreased with increasing age; however, it increased significantly in the oldest group and in highly degenerate discs.

We identified three matrix turnover phases. Phase I (growth) is characterized by active synthesis of matrix molecules and active denaturation of type II collagen. Phase II (maturation and ageing) is distinguished by a progressive drop in synthetic activity and a progressive reduction in denaturation of type II collagen. Phase III (degeneration and fibrotic) is illustrated by evidence for a lack of increased

Address correspondence to John Antoniou, Orthopaedic Research Laboratory, Royal Victoria Hospital, 687 Pine Avenue West, Room L4.68A, Montreal, Quebec, H3A 1A1, Canada. Phone: 514-842-1231; FAX: 514-843-1699; E-mail: janton@orpla.rvh.mcgill.ca. Address reprint requests to Mauro Alini, Orthopaedic Research Laboratory, Royal Victoria Hospital, 687 Pine Avenue West, Room L4.68A, Montreal, Quebec H3A 1A1, Canada. Phone: 514-842-1231; Fax: 514843-1699; E-mail: malini@orpla.ruh.mcgill.ca

Dr. Fred Nelson's present address is the Department of Orthopedics, Henry Ford Hospital, Detroit, Michigan 48202.

Dr. Anthony P. Hollander's present address is the Department of Human Metabolism and Clinical Biochemistry, University of Sheffield Medical School, Sheffield, UK.

Received for publication 12 April 1996 and accepted in revised form 19 June 1996.

J. Clin. Invest.

(C) The American Society for Clinical Investigation, Inc.

0021-9738/96/08/0996/08 \$2.00

Volume 98, Number 4, August 1996, 996-1003 synthesis of aggrecan and type II procollagen, but also by an increase in collagen type II denaturation and type I procollagen synthesis, both dependent on age and grade of tissue degeneration. (J. Clin. Invest. 1996. 98:996-1003.) Key words: aggrecan • collagens $\bullet$ matrix turnover $\bullet$ degradation $\bullet$ disc

\section{Introduction}

Degenerative disc disease is believed to begin as early as the second decade of life, and is viewed by most as an inevitable consequence of ageing. Despite its prevalence, there is no clear distinction between disc degeneration and normal maturation, nor is it clear why disc degeneration progresses slowly in some patients, whereas in others more rapid destruction of the intervertebral discs can occur.

The disc's low oxygen tension (1), low pH $(2,3)$, and relatively acellular environment (4) have brought into question the disc's ability to synthesize new matrix macromolecules to balance the process of degeneration (5). Evidence of a reparative process in the disc was first observed in animal studies that demonstrated partial regrowth after injection of chymopapain into the intervertebral discs $(6,7)$. More recently, this was indirectly observed in humans treated with chymopapain (8). However, little is known about the natural turnover of matrix components in the intervertebral disc with growth, ageing, and degeneration.

In recent years, knowledge of the basic molecular structure of cartilaginous tissues, mainly of articular cartilage, has grown rapidly (9-11), improving the ability to study matrix turnover at the molecular level. Immunoassays are now available to quantify tissue-specific matrix molecules, enabling investigations of turnover of matrix components in vivo (10-13). Such analyses may help identify the molecular basis of matrix degeneration in the intervertebral disc. However, when interpreting direct measurements of matrix macromolecules, it remains unclear whether they represent newly synthesized components, degradation products, or resident molecules. Recent work has led to the identification of specific epitopes or regions of molecules, recognizable or present only on degradation products of extracellular matrix proteins or on newly synthesized molecules. By immunoassay, this permits the distinction and quantification of matrix turnover changes (synthesis and degradation) within different tissues (14-16).

We have recently used some of these newly developed immunoassays to study human lumbar intervertebral disc matrix.

1. Abbreviations used in this paper: 846 , chondroitin sulfate epitope 846 in aggrecan; CPI (CPII), C-propeptide of type I (type II) procollagen; GAG, glycosaminoglycan. 
These techniques employ antibodies that recognize an epitope that is present on newly synthesized aggrecan (846), ${ }^{1}$ epitopes on the C-propeptides of newly synthesized type I and type II procollagens, and an epitope that is exposed, once type II collagen is cleaved and it loses its triple helical configuration (COL2-3/4m epitope). Together, the use of these assays permits an assessment of how disc matrix synthesis and degradation may vary with development, ageing, and degeneration.

The monoclonal antibody 846 recognizes a chondroitin sulfate epitope (17) on the largest aggrecan molecules showing $100 \%$ aggregability with hyaluronan (18). The epitope content is barely detectable in normal adult cartilage $(18,19)$, is increased in osteoarthritic cartilage (18), and is highest in fetal and neonatal cartilage $(18,19)$. In vitro studies have shown a direct correlation of the content of this epitope in cartilage to aggrecan synthesis, measured by incorporation of $\left.{ }^{35} \mathrm{~S}\right]$ sulfate into the glycosaminoglycans (GAG) of aggrecan (Jugessur, H., and A.R. Poole, unpublished observations). This suggests the 846 epitope can be used to identify newly synthesized aggrecan molecules.

The C-propeptides of type I (CPI) and type II (CPII) procollagens are released by the action of $\mathrm{C}$-proteases as these procollagens are incorporated into forming fibrils in the extracellular sites (20). The rate of synthesis of type II collagen, measured as ${ }^{3} \mathrm{H}$-proline incorporation into ${ }^{3} \mathrm{H}$-hydroxyproline, is proportional to the cartilage content of CPII, which has a half-life of 15-20 h (Nelson, F., and A.R. Poole; unpublished observations). The CPI content has previously been correlated to newly synthesized procollagen type I $(21,22)$. Both propeptides can be detected by specific immunoassays. A sandwich ELISA immunoassay specific for the quantification of CPI is commercially available (23), and an immunoassay for CPII was developed by Hinek et al. (24) and later modified (15).

A new immunochemical approach was developed for quantitative measurement of collagen damage by detecting denatured type II collagen (loss of triple helical configuration) (25). A monoclonal antibody (COL2-3/4m) was then developed that reacts with denatured, but not native, type II collagen (14). A quantitative method was also developed to proteolytically extract denatured and native collagen alpha-chains from cartilage. This immunoassay can therefore separately measure denatured and total type II collagen concentrations (14).

In this study, we have applied these new methods to measure newly synthesized proteoglycan aggrecan (846), newly synthesized type I and type II procollagens, and total and denatured type II collagen content in human lumbar intervertebral discs. These analyses have enabled us to identify distinct phases of disc matrix turnover related to growth, maturation and ageing, and degeneration.

\section{Methods}

Harvesting of intervertebral discs and method of sampling. 25 whole lumbar spine specimens were harvested from fresh cadavers ranging in age from $12 \mathrm{wk}$ to $79 \mathrm{yr}$ (mean of $29 \mathrm{yr}$ ). An 18-hr time-limit postmortem was set to minimize water content changes and enzymatic degeneration of the matrix. 121 intervertebral discs were examined. The number of spines obtained from each defined age group is shown in Table I. A complete medical and surgical history was obtained for each donor. Those with previous spine surgery, recent chemotherapy, radiation therapy to the spine, significant long-standing paralysis, an ankylosing process, or significant scolio/lordotic curvature (i.e., apex
Table I. Specimen Details

\begin{tabular}{lrrrrrrrrr}
\hline & \multicolumn{8}{c}{ Age range } \\
\cline { 2 - 9 } & $0-2$ & $2-5$ & $5-15$ & $15-25$ & $25-40$ & $40-60$ & $60-80$ & Total \\
\hline Total spines & 4 & 2 & 3 & 4 & 4 & 4 & 4 & 25 \\
Discs & & & & & & & & \\
Morphologic grade 1 & 20 & 10 & 15 & 10 & 2 & 0 & 0 & 57 \\
Morphologic grade 2 & 0 & 0 & 0 & 8 & 12 & 7 & 0 & 27 \\
Morphologic grade 3 & 0 & 0 & 0 & 2 & 6 & 6 & 13 & 27 \\
Morphologic grade 4 & 0 & 0 & 0 & 0 & 0 & 4 & 6 & 10 \\
Total discs & 20 & 10 & 15 & 20 & 20 & 17 & 19 & 121 \\
& & & & & & & & \\
\hline
\end{tabular}

of the curvature $>25^{\circ}$ ) were excluded from the study. The lumbar spines (T12 to S1) were dissected and retrieved. The specimens were vacuum sealed and then frozen at $-20^{\circ} \mathrm{C}$.

For analysis, these specimens were thawed and all segments were separated ( 5 per spine) by sawing transversely through the vertebral bodies. A 1-cm-thick midline sagittal slab was cut from all specimens with a fine, high speed circular saw (to avoid significant tissue loss). All specimens were graded for degeneration by an independent observer using the Thompson classification (26). This classification is a 5-category grading scheme for assessing gross morphology of midsagittal sections of human lumbar intervertebral disc. A photographic record was made of all slabs. The vertebral remnant was separated from the disc by scalpel dissection. 5 equal size $(10 \times 4 \times 7 \mathrm{~mm})$ specimens, representing the defined disc regions (i.e., anterior annulus, anterior intermediate zone, nucleus pulposus, posterior intermediate zone, and posterior annulus), were taken from each of the spine's 5 lumbar discs (L1-L2 to L5-S1). Each specimen was further cut into 3 equal size $(2 \times 4 \times 7 \mathrm{~mm})$ sections with a fine cutting block, providing 3 samples, each $\sim 50 \mathrm{mg}$ of tissue, from each region of the disc to be analyzed (see Fig. 1). These 3 samples underwent 1 of 3 biochemical protocols.

Determination of denatured and total type II collagen content. Added to these samples was $0.5 \mathrm{ml}$ of $1 \mathrm{mg} / \mathrm{ml}$ alpha-chymotrypsin in $50 \mathrm{mM}$ Tris, $\mathrm{pH} 7.6$ (with proteinase inhibitors: $1 \mathrm{mM}$ EDTA, $1 \mathrm{mM}$ iodoacetamide and $10 \mu \mathrm{g} / \mathrm{ml}$ pepstatin A) (Sigma Chemical Co., St. Louis, MO), to solubilize denatured type II collagen. Samples were
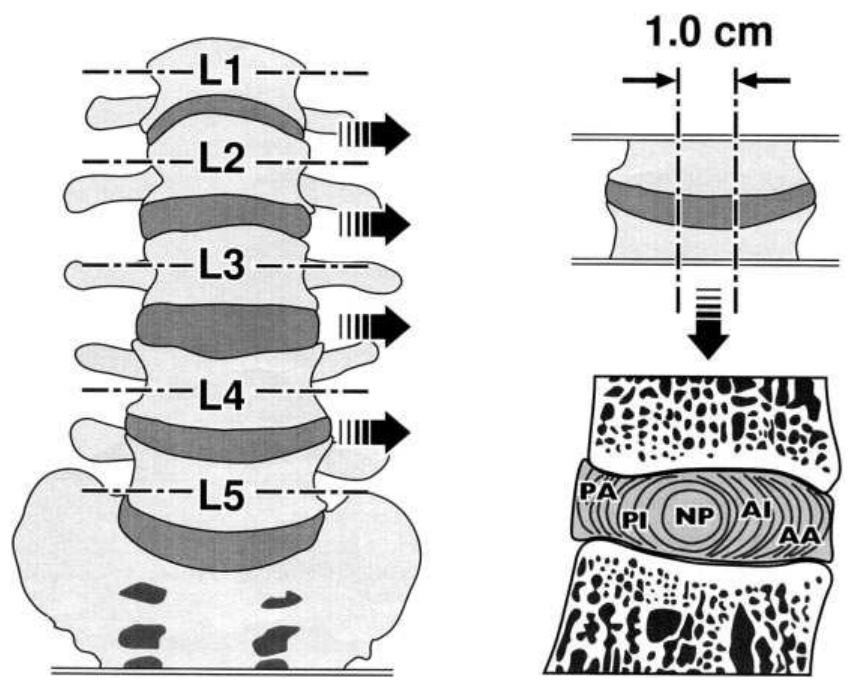

Figure 1. Schematic representation of the specimen isolation procedures. AA, anterior annulus; AI, anterior intermediate; NP, nucleus polposus; PA, posterior annulus; PI, posterior intermediate. 
incubated overnight at $37^{\circ} \mathrm{C}$. Then $200 \mu \mathrm{l}$ of TPCK $(N$-tosyl-phenylalanine-chloromethyl ketone) $(400 \mu \mathrm{g} / \mathrm{ml})$ were added to inhibit alpha-chymotrypsin activity, and the incubation was continued for 30 min at $37^{\circ} \mathrm{C}$. The disc residue was separated from the alpha-chymotrypsin extract by centrifugation, and remaining native collagen chains were digested with $0.5 \mathrm{ml}$ of $1 \mathrm{mg} / \mathrm{ml}$ proteinase $\mathrm{K}$ in the Tris buffer (containing the same proteinase inhibitors) overnight at $56^{\circ} \mathrm{C}$ (14). Tissue extracts were assayed for denatured collagen (alpha-chymotrypsin extract) and total type II collagen (proteinase K digestion) using an ELISA inhibition assay (14). The denatured and total type II collagen content of each sample was calculated from the concentration of alpha1(II)-CB11B epitope (14).

Determination of the contents of newly synthesized aggrecan proteoglycan (846 epitope) and procollagens type I and type II. These samples were extracted with $1 \mathrm{ml}$ of $4 \mathrm{M}$ guanidinium chloride in $50 \mathrm{mM}$ sodium acetate, $0.5 \%$ CHAPS (3-(3-cholamidopropyl) diethyl-ammonio-1 propanesulfonate), $\mathrm{pH} 5.8$ (with the following proteinase inhibitors: $10 \mu \mathrm{g} / \mathrm{ml}$ Pepstatin A, $1 \mathrm{mM}$ EDTA, $1 \mathrm{mM}$ iodoacetamide, and $1 \mathrm{mM}$ phenylmethylsulfonylfluoride), at $4^{\circ} \mathrm{C}$ for $48 \mathrm{~h}$ with agitation. The extract was dialyzed (mol wt cutoff 12,000-14,000) against 50 $\mathrm{mM}$ sodium acetate, $\mathrm{pH}$ 6.3. Aliquots of the dialyzed extracts were analyzed for their content of 846 epitope, C-propeptide of type I procollagen, and C-propeptide of type II procollagen.

The 846 epitope content was determined with a radioimmunoassay using fetal aggrecan as the standard (18). The CPI content was measured with a commercially available, sandwich ELISA immunoassay (Metra Biosystems, Inc., Palo Alto, CA) on aliquots of the guanidinium chloride extracts $(100 \mu \mathrm{l})$ after dialysis (23). For determination of the CPII content, a $25-\mu$ l solution of $1 \%$ CHAPS, $2 \mathrm{M}$ $\mathrm{NaCl}, 0.4 \mathrm{M}$ EDTA, $0.4 \mathrm{M}$ potassium acetate, $\mathrm{pH}$ 7.4, was added to aliquots of the guanidinium extracts $(75 \mu \mathrm{l})$ before assay (27). The antibody R160 ( $\operatorname{IgG})$ was incubated with the samples overnight. Antigen ${ }^{125}$ I-CPII was then added and allowed to compete with the cold antigen overnight. After centrifugation of the antibody complex (precipitated with protein A), the radioactivity in the pellet was measured. The standard was purified bovine CPII. For full details, see Hinek et al. (24).

Total water, collagen, and glycosaminoglycan content. Samples in this analysis were dried at $110^{\circ} \mathrm{C}$ for $4 \mathrm{~d}$ to obtain the dry weight. Hydroxyproline content was obtained from the dried tissues (28). Hydroxyproline content being equivalent to $10 \%$ of the weight of each collagen alpha chain, total collagen content per dry weight was estimated (29). The extracts derived from the alpha-chymotrypsin and proteinase $\mathrm{K}$ digestions were used for measurement of the total proteoglycan content as sulfated GAG content per dry weight with the dimethylmethylene blue dye binding colorimetric assay (30).

Statistical analyses. All values (mean \pm SEM) were recorded in a spreadsheet program according to age group, grade of degeneration, and disc region. The significance of ageing, location, and degeneration on the various matrix components was determined with ANOVA and multiple logistic regression analysis. Percentage of water content was correlated to GAG content and to percentage denatured type II collagen content in each region of the intervertebral disc with simple regression analysis.

\section{Results}

Table I shows the number of intervertebral discs obtained and their Thompson grade of degeneration. We found no significant differences (using multiple logistic regression analysis), in the concentrations of the components being assessed, in discs with the same grade of degeneration, taken from different segmental levels (L1-L2 to L5-S1) of the same spine (no intraspine variation). This was also true of discs from different spines of the same age group and the same Thompson grade (no interspine variation). No differences were found between

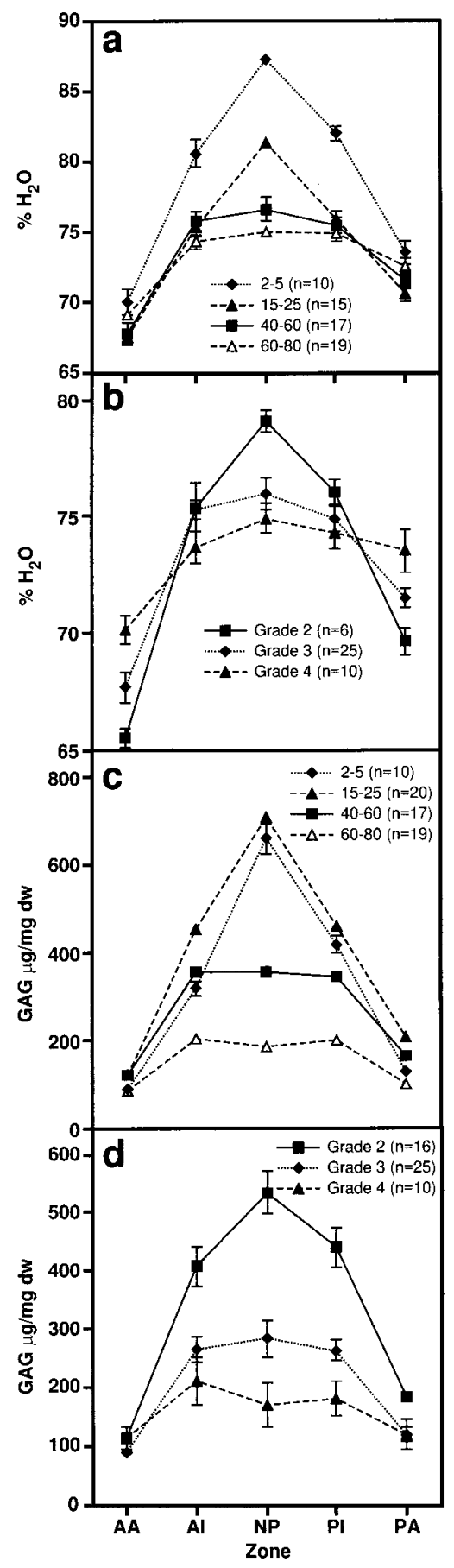

Figure 2. Changes in water content $(a, b)$ and GAG content $(c, d)$ related to age $(a, c)$ and grade $(b, d)$ within disc zones. dw, dry weight.

sexes (female, $n=11$; male, $n=14$ ). Therefore, our results are presented as mean values \pm SEM for all discs within a defined age group, location, and Thompson grade.

\section{Matrix component changes}

Water and glycosaminoglycan content. In donors up to $25 \mathrm{yr}$, water content was significantly higher in the nucleus, decreasing progressively toward the annulus (Fig. $2 a$ ). With maturation and ageing, water content dropped significantly, particularly in the nucleus, and the difference between the nucleus and annulus became less marked. In addition, water content in the nucleus was significantly reduced $(n=46, P<0.001)$ with increasing grade of degeneration in the $>30 \mathrm{yr}$ population 
(Fig. $2 b$ ). Whereas, when the water content dropped in the nucleus pulposus, it increased in the annulus with increasing grade (Fig. $2 b$ ). Similar to water content, GAG content was significantly $(P<0.001)$ higher in the nucleus than in the annulus in the population up to $25 \mathrm{yr}$ (Fig. $2 \mathrm{c}$ ). With ageing, there was a marked drop that was most apparent in the nucleus pulposus (Fig. $2 \mathrm{c}$ ). In the oldest age group (60-80 yr), GAG content was similar in all disc regions (Fig. $2 c$ ). GAG content was also significantly reduced in the nucleus and, to a lesser degree, in the intermediate zones, with increasing grade of degeneration in discs from the population $>30 \mathrm{yr}$ (Fig. $2 d$ ). More important, a strong correlation was noted between water and GAG contents $(r=0.693, P<0.0001 ; n=71)$.

Total and type II collagen content. Independent of age, total collagen content per dry weight (expressed as hydroxyproline content) was consistently higher $(P<0.001)$ in the annulus and intermediate zones than in the nucleus (Fig. $3 a$ ). Content in the annulus and intermediate zones decreased significantly with increasing age, but remained unchanged in the nucleus pulposus (Fig. $3 a$ ). Assuming the hydroxyproline content of fibrillar collagens represents about $10 \%$ of the weight of each alpha chain (29), type II collagen (by immunoassay) accounted for practically all the collagen in the nucleus of

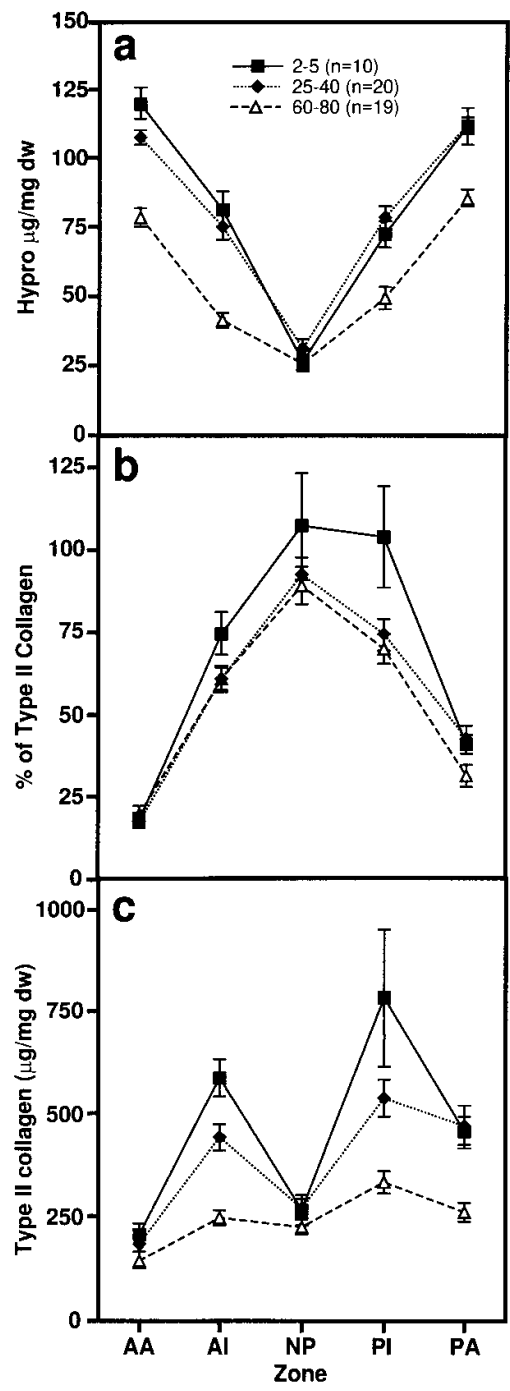

Figure 3. Hydroxyproline content $(a)$, percentage of type II collagen content $(b)$, and absolute type II collagen content $(c)$ related to age within disc zones. The key in $a$ is applicable to $b$ and $c$ also. dw, dry weight. those up to $5 \mathrm{yr}$ (Fig. $3 \mathrm{~b}$ ). With age, the percentage of type II collagen in the nucleus dropped to about $90 \%$ of the total collagen content, whereas it remained relatively constant in the annulus; about $20 \%$ in the anterior annulus and $35 \%$ in the posterior annulus (Fig. $3 \mathrm{~b}$ ). The absolute concentration of type II collagen in the disc showed changes that depended on age and region. Up to $40 \mathrm{yr}$, type II collagen content in both intermediate zones was higher than in the nucleus (Fig. $3 c$ ). With increasing age, the content dropped in the intermediate zones and in the posterior annulus. In the oldest age group (60-80), there was no difference between regions (Fig. 3c). In
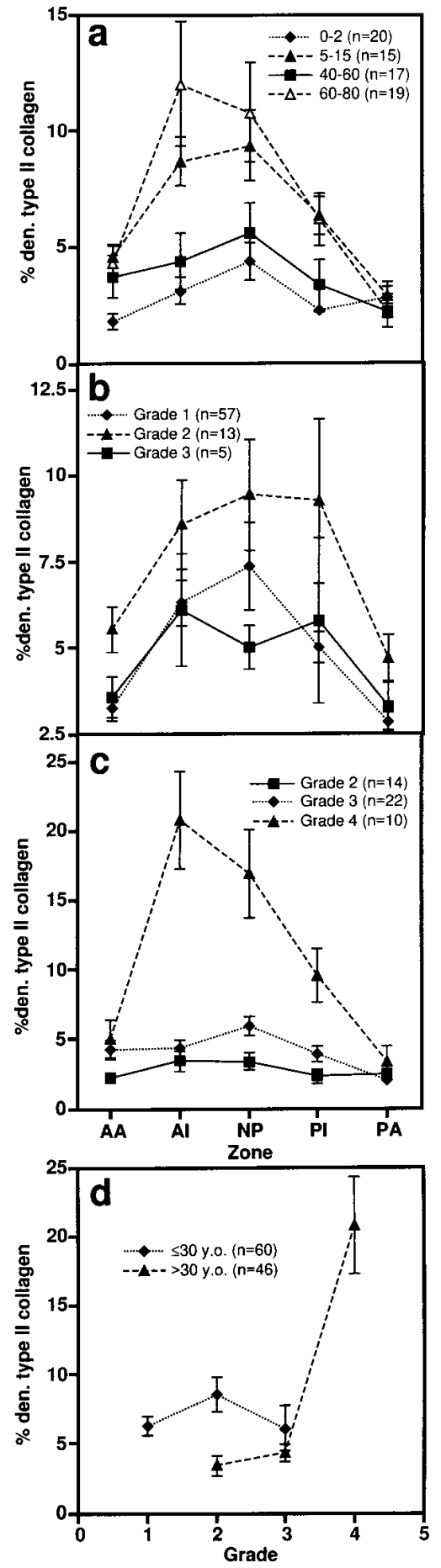

Figure 4. Percentage of denatured type II collagen related to age $(a)$ and morphological grade $(b, c)$ in two groups separated at 30 yr: $\leq 30 \mathrm{yr}(b)$ and $>30$ yr $(c)$. Percentage of denatured type II collagen content of the nucleus pulposus vs morphologic grade in two groups separated at $30 \mathrm{yr}(d)$. 
the anterior annulus and nucleus regions, type II collagen content was unchanged with age (Fig. $3 c$ ). Unlike GAG content, there was no significant correlation between total collagen content and grade of degeneration (data not shown).

\section{Matrix synthesis and degradation}

Percentage of denatured type II collagen. The proportion of denatured type II collagen varied with age and location (Fig. $4 a$ ). It was lowest in the neonatal age group (0-2 yr), with no significant difference between the disc regions (Fig. $4 a$ ). In the 2-5 (data not shown) and 5-15 yr age groups, percentages significantly increased in all regions except the posterior annulus (Fig. $4 a$ ). In the older age groups (up to $60 \mathrm{yr}$ ) percentages dropped, approaching those in the $0-2$ yr age group (Fig. $4 a$ ). However, in the oldest group (60-80 yr), type II collagen denaturation was again significantly increased in the nucleus and intermediate regions (Fig. $4 a$ ). Figs. $4, b$ and $c$ show the percentage of denatured type II collagen correlated to gross morphologic Thompson grade in populations $<30$ (Fig. $4 \mathrm{~b}$ ) and $>30 \mathrm{yr}$ (Fig. $4 c$ ). Though not statistically significant, the percentage of denatured type II collagen tended to be higher in the population $<30$ yr with grade II degeneration compared to grades I and III (Fig. $4 b$ ). Percentages were highest in the nucleus and intermediate zones (Fig. 4 b). By contrast, percentages were markedly increased in the population $>30 \mathrm{yr}$ with grade IV degeneration, whereas grades II and III were similar (Fig. 4c). The amount of denatured type II collagen in grade IV discs reached $15-20 \%$ of the total collagen in the nucleus and anterior intermediate zones, but was essentially unchanged in the annulus. This marked increase contrasted with the $5-10 \%$ denaturation observed during growth (Fig. $4 a$ ). Fig. $4 d$ further summarizes the increase in denatured type II collagen in the nucleus pulposus with ageing that was dependent on the degeneration (grade). This contrasted with that observed during growth, which was independent of degeneration. Comparing water content to the percentage of denatured type II collagen, there was a weak inverse relationship, most apparent in the nucleus and intermediate zones in the $>30$ population $(r=$ $0.375, P<0.01, n=46)$.

Levels of 846 epitope of proteoglycan aggrecan, and the propeptides of procollagens I and II. Levels of 846 and CPII epitopes dropped significantly $(P<0.001)$ with increasing age (Fig. 5, $a$ and $b$ ). These levels were highest in the nucleus, and lower in the intermediate zones of the neonatal (data not shown) and 2-5-yr age groups (Fig. 5, $a$ and $b$ ). Both 846 and CPII levels had dropped markedly by $5-15 \mathrm{yr}$ and continued to drop in all regions thereafter, being least pronounced in the annulus (Fig. 5, $a$ and $b$ ), although low levels were still detectable in the older age groups. The 846 and CPII levels also significantly $(P<0.001)$ decreased with increased gross morphologic grade of discs in the $>30$ age group (Fig. 5, $c$ and $d$ ). This was particularly evident in the nucleus and intermediate zones.

Interestingly, no significant difference in CPI levels was observed between the different regions of the disc (data not shown). The total disc CPI level was highest in the neonatal and $2-5 \mathrm{yr}$ age groups (Fig. $6 a$ ). In contrast to the 846 and CPII levels, which remained relatively high (Fig. 5, $a$ and $b$ ), the CPI level dropped to its lowest in the 5-15 yr age group (Fig. 6a), and then showed a small but steady increase with increasing age (Fig. $6 a$ ). In the population $>30$, CPI level increased significantly $(n=46, P<0.01)$ in the most degenerate discs (grade IV), reaching levels in grade I discs $<30$ yr (Fig. $6 b$ ).

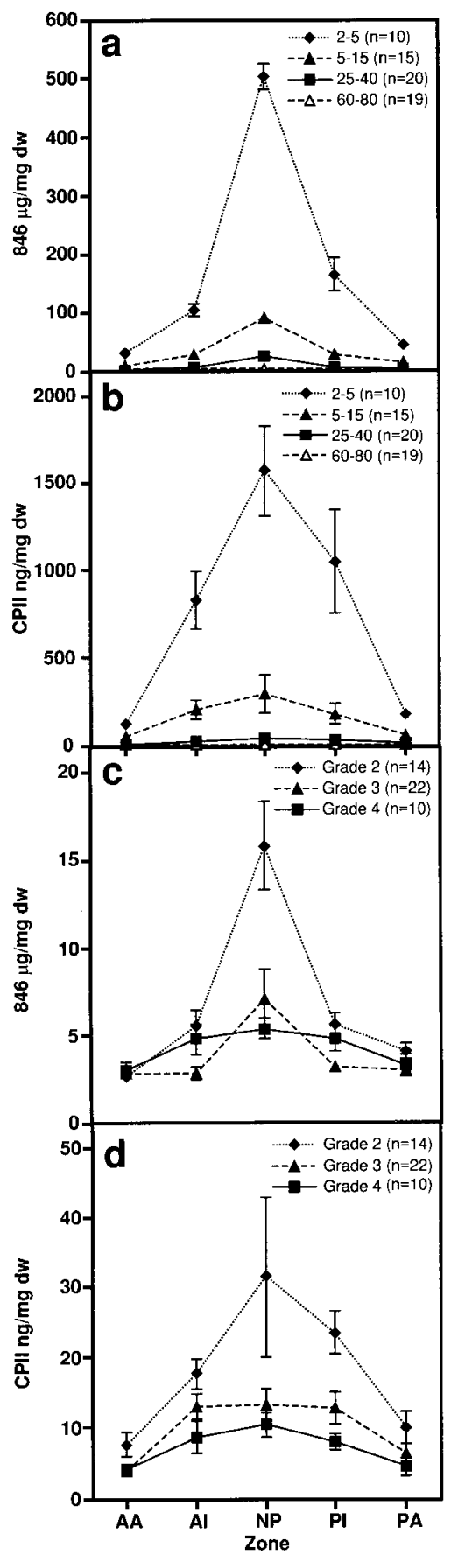

Figure 5. Changes in 846 epitope content $(a$, $c)$ and CPII $(b, d)$ content related to age $(a, b)$ and morphological grade $(c, d)$ within disc zones. Data are plotted with increasing morphologic grade in those $>30$ yr. dw, dry weight.

\section{Discussion}

The matrix of the intervertebral disc comprises mainly a fibrillar collagen network that offers tensile strength $(31,32)$ and aggregating proteoglycans that resist compressive forces. These major components form a mesh suited for containing water molecules, especially in the nucleus. An intact extracellular matrix is essential to normal disc function. The ability of disc tissue to withstand mechanical forces largely depends on the structural integrity of the matrix and on the physiological balance of collagen, proteoglycan, and water content. The continuing debate on disc nutrition, and the highly acellular nature of the disc, has raised questions as to whether the disc suf- 

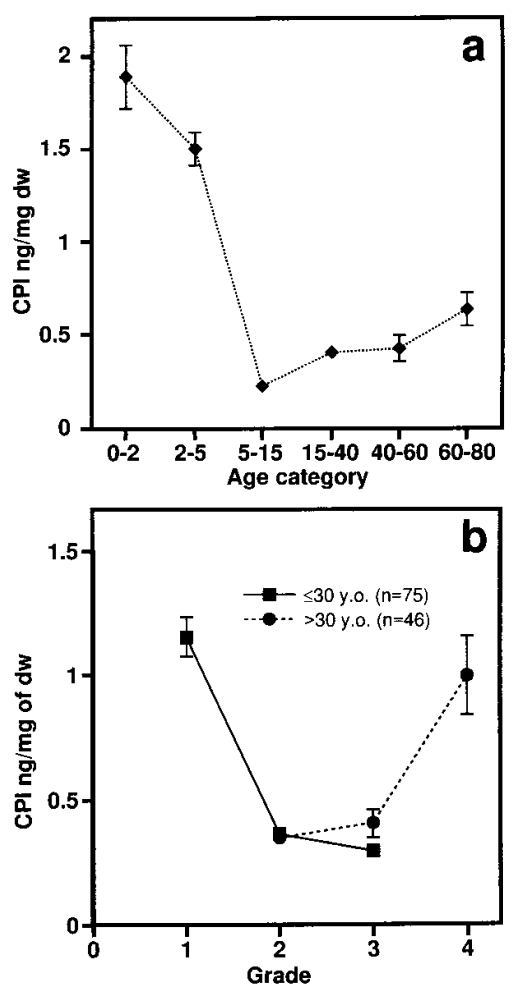

Figure 6. CPI content in whole disc related to age (a). Type I C-propeptide content vs morphologic grade for two age populations separated at $30 \mathrm{yr}$ (b). dw, dry weight.

fers damage to its collagen molecules and whether it can generate new collagen and proteoglycan in a reparative fashion during ageing and degeneration, as in osteoarthritic cartilage $(5,33)$.

Current knowledge of the pathophysiology of tissue destruction, and the macromolecular changes in the matrix of the human intervertebral disc with ageing and degeneration, has been based mainly on studies analyzing the total content of the different matrix components. With the advent of new technology used here to study type II collagen denaturation, we now know that there is increased damage to collagen fibrils in normal adult discs compared with hyaline cartilage (34). Recent studies of human discs have also revealed increased cleavage of link protein (35) accompanying the loss of aggrecan aggregation (36) and an overall reduction in the content of these molecules with age (35-38). However, these studies did not distinguish between growth, maturation, ageing, and disc degeneration. Moreover, they did not differentiate between newly synthesized components, degradation products, and resident molecules.

In vivo metabolic studies performed on animals have mainly examined ${ }^{35} \mathrm{SO}_{4}{ }^{2-}$ incorporation into newly synthesized proteoglycan (aggrecan) at different intervals after injection of radiolabeled materials (39-43). Such experiments cannot be done in humans and the results do not necessarily apply. Furthermore, no study has investigated collagen metabolism (synthesis and degradation), nor variations of general cellular biosynthesis in the different regions of the human intervertebral disc with ageing and degeneration.

In vivo studies of human intervertebral disc metabolism are needed to understand the synthetic and degradative processes occurring during development, ageing, and degeneration. In this study, we introduce the use of newly developed immunoassays to study matrix synthesis and degradation. Although the half-lives of the 846 epitope on aggrecan and CPII were not determined in disc tissue, and may not be the same as in cartilage because of differences in anatomy and hence of diffusion pathways, the use of these epitopes permits new insight into disc metabolism that would not otherwise be possible. Using these and other new techniques, we have obtained the first evidence pointing to significant changes in collagen and proteoglycan synthesis and collagen denaturation related to location, development, ageing, and degeneration of the human lumbar disc.

With increasing age and grade of degeneration, the very high levels of 846 and CPII epitopes in the nucleus pulposus of the very young drop markedly and equilibrate with the levels in the intermediate zone. The high synthetic activity in the nucleus could be secondary to a higher synthetic activity per cell. It could also be the result of deposition of newly synthesized matrix components derived from cells of the intermediate zone. This process has been likened to a "filling in" of the nucleus pulposus $(6,7)$. This interpretation has been made because of the low cellularity and reduced cellular activity previously noted in the nucleus pulposus, compared with the relatively higher and more active cellular content in the inner annular region $(6,7)$. The indicated and parallel drop in synthetic activity with increasing age of both these molecules could also be related to disc nutrition, which is essential to preserving the role of disc cells in matrix assembly and resorption. There are two nutrition exchange routes between the discs and the surrounding tissues: blood vessels in the outer one third of the annulus fibrosus, and vascular channels in the cartilage end-plates (44). In persons from the fourth decade on, no vascular structures are detected in the annulus (45). With increasing age, end-plate vessels may no longer function because of a decrease in tissue permeability (1). Reduced circulation within the disc with ageing and degeneration could therefore be partly responsible for the marked decrease in synthetic activity. In fact, a decrease in disc nutrition has been shown to induce a drop in the disc's $\mathrm{pH}(2,3)$. In such an environment, disc tissues have shown diminished proteoglycan synthesis (46).

Although type I procollagen synthesis drops similarly to aggrecan and type II procollagen with development and ageing, unlike aggrecan and type II collagen it does not vary significantly between the different regions of the disc. In the youngest age groups, the disc's average CPI content is about 1,000 times less than its CPII content. With the considerable decrease in CPII content and progressive increase in CPI content, this ratio drops to only 10 -fold in the older age groups. However, CPI content increases significantly in most degenerated discs (grade IV). This reversal suggests independent regulatory mechanisms in the synthesis of these collagens during ageing compared with degeneration.

Degradative changes in type II collagen that may occur with development, maturation, ageing, and degeneration were studied with a newly developed method (14), allowing us to measure the total content of type II collagen and the percentage of denatured type II collagen. The denaturation of type II collagen in discs, independent of grade, from the 2-5-yr age group was markedly higher than that of the $0-2-y r$ age group. This indicates significant cleavage of this molecule as part of growth and development. With further growth, the amount of denatured collagen II decreased as did synthesis of this molecule. It was only when degeneration developed in the adult that the high levels of denaturation seen in the juvenile were again observed; this time not accompanied by active synthesis of type II procollagen. Despite the marked suppression of syn- 
thesis with ageing and degeneration, it is important to note that some matrix synthesis continued in the older age groups. These synthetically active cells may therefore, by means of a biological factor or a combination of factors, be susceptible to being stimulated to increase their synthetic activity to partially restore their surrounding matrix molecules toward normal physiological levels.

In this study, we have also related the content of total collagen and type II collagen to age, location, and degeneration. The two major collagens (types I and II) in the intervertebral disc are said to be distributed radially in opposing concentration gradients: type II collagen being the major constituent of the nucleus pulposus, and type I collagen of the annulus fibrosus (47). However, the actual content of each collagen was previously unknown in the human disc. Previous work $(47,48)$ has revealed that there is a markedly lower total collagen content in the nucleus than in the annulus. With increasing age, total collagen content decreases in the annulus but not in the nucleus. In our studies, no relationship was found between total collagen content or the absolute content of type II collagen and the morphologic grade of disc degeneration. We show that type II collagen represents the majority of total collagen in the nucleus and a significant proportion of total collagen in the annulus (up to $40 \%$ ). The concentration of type II collagen was surprisingly at its highest in the intermediate zones and dropped with increasing age.

Chemical analysis previously has shown evidence of a GAG concentration gradient that increases from the outer annulus to the central portion of the nucleus $(49,50)$. Our observations corroborate and further demonstrate that this distribution is true in all age groups. In addition, GAG content decreased with ageing, from the neonate to the oldest age group, in all regions of the disc. This decrease was most evident in the nucleus. Whereas the younger population exhibited high GAG content independent of grade, GAG content was inversely correlated to gross morphologic grade in the $>30$ age group. These observations clearly distinguish between the morphologic appearance and the state of the matrix in comparisons of the younger and older populations.

A drop in water content of the disc related to age, location, and degeneration had been shown (51). We have corroborated previous work that showed the most significant drop in water content with age occurred in the nucleus pulposus (51). MRI studies attributed the loss of T2 weighted image brightness in degenerated discs to a decrease in the water content (38). We describe a similar drop in water content with increased gross morphologic grade in a population $>30$ (no such trend was present in the younger population). The peripheral redistribution of water with increased gross morphologic grade may be a real phenomenon, or it may be the result of water redistribution after freezing and thawing of specimens. Since, however, our data are expressed per dry weight, any difference in water content would not influence our final conclusions with respect to changes in collagen and proteoglycan related to synthesis and degradation. Further investigation may be necessary to discriminate between the two.

In these studies, we may conclude that we have identified three phases of matrix turnover related to age and grade of degeneration by correlating matrix biosynthetic marker levels to the disc's denatured type II collagen content. These are the growth, maturation and ageing, and degeneration and fibrotic phases (summarized in Fig. 7). Phase I, growth period (0-15 $\mathrm{yr}$ ) is indicative of active synthesis of aggrecan (846 epitope)

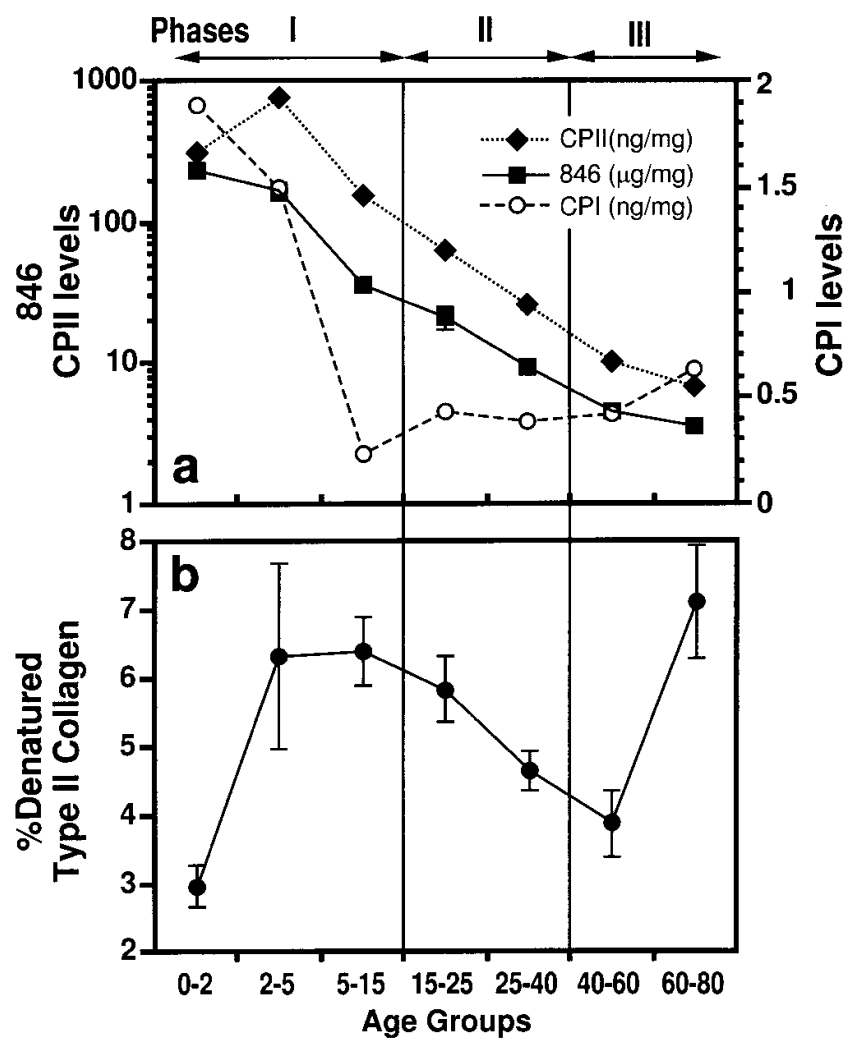

Figure 7. Summary of evidence for synthetic changes $(a)$ and degradative changes (denatured type II collagen) $(b)$ with age in whole disc. The SEM is included within the symbols because of the semi-log scale in $a$.

and procollagens I and II, along with increased growth-associated denaturation type II collagen (2-15 yr). These processes are independent of disc morphologic grade (growth phase). Phase II (15-40 yr) is distinguished by a reduction in the synthesis of cartilage molecules except for type I procollagen (apparent at 5-15 yr), along with a reduction in denatured type II collagen (ageing and maturation phase). Phase III (40-80 yr) shows an increase in denatured type II collagen at 60-80 yr, dependent on grade, along with a small increase in synthesis of type I procollagen, but no increase in type II procollagen or aggrecan synthesis (degeneration and fibrotic phase).

Identification of these phases improves our understanding of disc growth, maturation, normal ageing, and degeneration of this tissue. This brings us one step closer to being able to control matrix turnover with the purpose of containing or preventing human intervertebral disc degeneration.

\section{Acknowledgments}

The excellent technical work of N.M. Goudsouzian, BSc., and T.F. Heathfield, BSc., is gratefully acknowledged. We also thank Quebec Transplant and the pathology departments of the Montreal General Hospital (Dr. W.P. Duguid), Royal Victoria Hospital (Dr. B. Case), and Saint-Justine Hospital (Dr. J. Michaud) for their invaluable help in obtaining the spines, Aejaz Zahid for his assistance in preparing the tissue, Jane Wishart and Mark Lepik who prepared the figures, and Lorne Beckman for editing.

This study was funded by research grants from the Arbeitsgemeinschaft für Osteosynthesefragen/Association for the Study of the 
Internal Fixation foundation, Shriners of North America, Medical Research Council of Canada, Pfizer Central Research, Monsanto (to R.A. Poole), and Arthritis Society of Canada (to A.P. Hollander).

\section{References}

1. Holm, S., A. Maroudas, J.P.G. Urban, G. Sestam, and A. Nachemson. 1981. Nutrition of the intervertebral disc: somite transport and mechanism. Connect. Tissue Res. 8:101-108.

2. Diamant, B., J. Karlsson, and A. Nachemson. 1968. Correlation between lactate levels and $\mathrm{pH}$ in disc of patients with lumbar rhizopathies. Experientia (Basel). 24:1195-1196.

3. Nachemson, A. 1969. Intradiscal measurements of $\mathrm{pH}$ in patients with lumbar rhizopathies. Acta Orthop. Scand. 40:23-42.

4. Oegema, T.J. 1993. Biochemistry of the intervertebral disc. Clin. Sports Med. 12:419-439.

5. Buckwalter, J.A. 1995. Spine update: aging and degeneration of the human intervertebral disc. Spine. 20:1307-1314.

6. Bradford, D.S., T.J. Oegema, M. Cooper, K. Wakano, and E.Y. Chao. 1984. Chymopapain, chemonucleolysis, and nucleus pulposus regeneration. A biochemical and biomechanical study. Spine. 9:135-147.

7. Garvin, P.J., and R.B. Jennings. 1973. Long-term effects of chymopapain on intervertebral disks of dogs. Clin. Orthop. Relat. Res. 92:281-295.

8. Hadjipavlou, A., P. Lander, and J. Antoniou. 1992. The effect of chymopapain on low back pain. Orthop. Rev. 21:733-738.

9. Heinegård, D., and Å. Oldberg. 1993. Glycosylated matrix proteins. In Connective Tissue and Its Heritable Disorders. P.M. Rayce and B. Steinmann, editors. Wiley-Liss Inc., New York. 189-209.

10. Poole, A.R. 1994. Immunochemical markers of joint inflammation, skeletal damage and repair. Where are they now? Ann. Rheum. Dis. 53:3-5.

11. Von der Mark, K., and S. Goodman. 1993. Adhesive glycoproteins. In Connective Tissue and Its Heritable Disorders. P.M. Royce and B. Steinmann, editors. Wiley-Liss Inc., New York. 211-236.

12. Heinegård, D., and T. Saxne. 1991. Molecular markers of processes in cartilage in joint disease. Br. J. Rheumatol. 30(Suppl. 1):21-24.

13. Thonar, E.J.M.A., A. Shinmei, and L.S. Lohmander. 1993. Body fluid markers of cartilage changes in osteoarthritis. Rheum. Dis. Clin. N. Am. 19:635-657.

14. Hollander, A.P., T.F. Heathfield, C. Webber, Y. Iwata, R. Bourne, C. Rorabeck, and A.R. Poole. 1994. Increased damage to type II collagen in osteoarthritic articular cartilage detected by a new immunoassay. J. Clin. Invest. 93:1722-1732.

15. Mansson, B., D. Carey, M. Alini, M. Ionescu, L.C. Rosenberg, A.R. Poole, D. Heinegård, and T. Saxne. 1995. Cartilage and bone metabolism in rheumatoid arthritis: differences between rapid and slow progression of disease identified by serum markers of cartilage metabolism. J. Clin. Invest. 95:1071-1077.

16. Antoniou, J., N.M. Goudsouzian, T.F. Heathfield, N. Winterbottom, T. Steffan, A.R. Poole, M. Aebi, and M. Alini. 1996. The human lumbar endplate: evidence for changes in the biosynthesis and denaturation of the extracellular matrix with growth, maturation, ageing and denaturation. Spine. 21:1153-1161.

17. Caterson, B., J.E. Christner, J.R. Baker, and J.R. Couchman. 1985. Production and characterization of monoclonal antibodies directed against connective tissue proteoglycans. Fed. Proc. 44:386-393.

18. Rizkalla, G., A. Reiner, E. Bogoch, and A.R. Poole. 1992. Studies of the articular cartilage proteoglycan aggrecan in health and osteoarthritis. Evidence for molecular heterogeneity and extensive molecular changes in disease. J. Clin. Invest. 90:2268-2277.

19. Glant, T.T., K. Mikecz, P.J. Roughley, E. Buzas, and A.R. Poole. 1986. Age-related changes in protein-related epitopes of human articular cartilage proteoglycans. Biochem. J. 236:71-75.

20. Poole, A.R. 1993. Cartilage in health and disease. In Arthritis and Allied Conditions. 12th ed. D.J. McCarthy and W.J. Koopmann, editors. Lea \& Febiger, Philadelphia, PA. 279-333.

21. Parfitt, A.M., L.S. Simon, A.R. Villanueva, and S.M. Krane. 1987. Procollagen type I carboxy-terminal extension peptide in serum as a marker of collagen biosynthesis in bone. J. Bone Miner. Res. 2:427-436.

22. Prokop, D.J., K.I. Kivirikko, L. Tuderman, and N.A. Guzman. 1979. The biosynthesis of collage and its disorders. N. Engl. J. Med. 301:13-23.

23. Winterbottom, N., S. Vernon, K. Freeman, G. Daniloff, and S. Seyedin. 1992. An immunoassay for the C-terminal propeptide of type I collagen. $J$. Bone Miner. Res. 7(Suppl 1):254.

24. Hinek, A., A. Reiner, and A.R. Poole. 1987. The calcification of cartilage matrix in chondrocyte culture: studies of the C-propeptide of type II collagen (chondrocalcin). J. Cell Biol. 104:1435-1441.

25. Dodge, G.R., and A.R. Poole. 1989. Immunohistochemical detection and immunological analysis of type II collagen degradation in human normal, rheumatoid and osteoarthritic articular cartilages and explants of bovine articular cartilage cultured with interleukin-1. J. Clin. Invest. 83:647-661.

26. Thompson, J.P., R.H. Pearce, M.T. Schechter, M.E. Adams, I.K. Tsang, and P.B. Bishop. 1990. Preliminary evaluation of a scheme for grading the gross morphology of the human intervertebral disc. Spine. 15:411-415.

27. Alini, M., Y. Matsui, G.R. Dodge, and A.R. Poole. 1992. The extracellular matrix of cartilage in the growth plate before and during calcification: changes in composition and degradation of type II collagen. Calcif. Tissue Int. 50:327-335.

28. Burleigh, M.C., A.J. Barrett, and G.S. Lazarus. 1974. Cathepsin B1. A lysosomal enzyme that degrades native collagen. Biochem. J. 137:387-398.

29. Nimni, M.E. 1983. Collagen: structure, function, and metabolism in normal and fibrotic tissues. Semin. Arthritis Rheum. 13:1-86.

30. Farndale, R.W., D.J. Buttle, and A.J. Barrett. 1986. Improved quantitation and discrimination of sulphated glycosaminoglycans by use of dimethylmethylene blue. Biochim. Biophys. Acta. 883:173-177.

31. Kempson, G.E., H. Muir, C. Pollard, and M. Tuke. 1973. The tensile properties of the cartilage of human femoral condyles related to the content of collagen and glycosaminoglycans. Biochim. Biophys. Acta. 297:465-472.

32. Schmidt, M.D., V.C. Mow, L.E. Chun, and D.R. Eyre. 1990. Effects of proteoglycan extraction on the tensile behavior of articular cartilage. J. Orthop. Res. 8:353-363.

33. Aigner, T., W. Bertling, H. Stoss, G. Weseloh, and K. von der Mark. 1993. Independent expression of fibril-forming collagens I, II, and III in chondrocytes of human osteoarthritic cartilage. J. Clin. Invest. 91:829-837.

34. Hollander, A.P., T.F. Heathfield, J.J. Liu, I. Pidoux, P.J. Roughley, J.S. Mort, and A.R. Poole. 1996. Enhanced denaturation of the alpha1(II) chains of type II collagen in normal adult human intervertebral discs compared to femoral articular cartilage. J. Orthop. Res. 14:61-66.

35. Pearce, R.H., J.M. Mathieson, J.S. Mort, and P.J. Roughley. 1989. The effect of age on the abundance and fragmentation of link protein of the human intervertebral disc. J. Orthop. Res. 7:861-867.

36. McDevitt, C.A. 1988. Proteoglycans of the intervertebral disc. The biology of the intervertebral disc. Vol 1. P. Ghosh, editor. CRC Press Inc, Boca Raton, FL. 151-170.

37. Pearce, R.H., J.G. Beverley, and M.E. Adams. 1987. Degeneration and the chemical composition of the human lumbar intervertebral disc. J. Orthop. Res. 5:198-205.

38. Pearce, R.H., J.P. Thompson, G.M. Bebault, and B. Flak. 1991. Magnetic resonance imaging reflects changes of ageing degeneration in the human intervertebral disc. J. Rheumatol. 18(Suppl. 27):42-43.

39. Cole, T.C., D. Burkhardt, L. Frost, and P. Ghosh. 1985. The proteoglycans of the canine intervertebral disc. Biochim. Biophys. Acta. 839:127-138.

40. Cole, T.C., P. Ghosh, and T.F.K. Taylor. 1986. Variation of the proteoglycans of the canine intervertebral disc with ageing. Biochim. Biophys. Acta. 880:209-219.

41. Lohmander, S., C.A. Antomopoulus, and U. Freiberg. 1973. Chemical and metabolic heterogeneity of chondroitin sulphate and keratan sulphate in guinea pig cartilage and nucleus pulposus. Biochim. Biophys. Acta. 304:430448

42. Venn, G., and R.M. Mason. 1983. Biosynthesis and metabolism in vivo of intervertebral disc proteoglycans in the mouse. Biochem. J. 215:217-225.

43. Venn, G., and R.M. Mason. 1986. Changes in mouse intervertebral disc proteoglycan synthesis with age: hereditary kyphoscoliosis is associated with elevated synthesis. Biochem. J. 234:475-479.

44. Moore, R.J., B. Vernon-Roberts, and R.D. Fraser. 1992. Changes in the endplate vascularity after an outer annulus tear in the sheep. Spine. 17:874-878.

45. Rudert, M., and B. Tillmann. 1993. Lymph and blood supply of the human intervertebral disc. Acta Orthop. Scand. 64:37-40.

46. Ohshima, H., and J.P.G. Urban. 1992. The effect of lactate and $\mathrm{pH}$ on proteoglycan and protein synthesis rates in the intervertebral disc. Spine. 17: 1079-1082.

47. Eyre, D., and H. Muir. 1976. Types I and II collagens in intervertebral disc. Interchanging radial distributions in annulus fibrosus. Biochem. J. 157: 267-270.

48. Eyre, D., and H. Muir. 1977. Quantitative analysis of type I and type II collagens in human intervertebral discs at various ages. Biochim. Biophys. Acta. $29-42$.

49. Bushell, G.R., P. Ghosh, T.F.K. Taylor, and W.H. Akeson. 1977. Proteoglycan chemistry of intervertebral discs. Clin. Orthop. Relat. Res. 129:115123.

50. Urban, J.P.G., and A. Maroudas. 1979. The measurement of fixed charge density in the intervertebral disc. Biochim. Biophys. Acta. 586:166-178.

51. Gower, W.E., and V. Pedrini. 1969. Age-related variations in proteinpolysaccharide from human nucleus pulposus, annulus fibrosus and costal cartilage. J. Bone J. Surg. Am. Vol. 51:1154-1159. 